\title{
Dissection of structural dynamics of chromatin fibers by single-molecule magnetic tweezers
}

\author{
Xue Xiao $^{1,3}$, Liping Dong ${ }^{2,3}$, Yi-Zhou Wang ${ }^{1,3}$, Peng-Ye Wang ${ }^{1,3}$, \\ Ming $\mathrm{Li}^{1,3}$, Guohong $\mathrm{Li}^{2,3}$, Ping Chen ${ }^{2 \bowtie}$, Wei $\mathrm{Li}^{1 \bowtie}$ \\ 1 National Laboratory for Condensed Matter Physics and Key Laboratory of Soft Matter Physics, Institute of Physics, \\ Chinese Academy of Sciences, Beijing 100190, China \\ 2 National Laboratory of Biomacromolecules, CAS Center for Excellence in Biomacromolecules, Institute of \\ Biophysics, Chinese Academy of Sciences, Beijing 100101, China \\ 3 University of Chinese Academy of Sciences, Beijing 100049, China
}

Received: 26 June 2018 / Accepted: 18 July 2018 / Published online: 29 August 2018

\begin{abstract}
The accessibility of genomic DNA, as a key determinant of gene-related processes, is dependent on the packing density and structural dynamics of chromatin fiber. However, due to the highly dynamic and heterogeneous properties of chromatin fiber, it is technically challenging to study these properties of chromatin. Here, we report a strategy for dissecting the dynamics of chromatin fibers based on singlemolecule magnetic tweezers. Using magnetic tweezers, we can manipulate the chromatin fiber and trace its extension during the folding and unfolding process under tension to investigate the dynamic structural transitions at single-molecule level. The highly accurate and reliable in vitro single-molecule strategy provides a new research platform to dissect the structural dynamics of chromatin fiber and its regulation by different epigenetic factors during gene expression.
\end{abstract}

Keywords Magnetic tweezers, Chromatin fiber, Dynamics, Single molecule

\section{INTRODUCTION}

In eukaryotic cells, genomic DNA is wrapped on histones to form the nucleosome (Richmond and Davey 2003), which is the basic repeating unit of chromatin, and then further folds to condensed chromatin fibers (Bickmore and van Steensel 2013). But the transcription and replication of DNA require the accessibility of DNA double-helix structure by transcription factors and polymerases. The packing density and structural dynamics of chromatin fibers are regulated by many epigenetic factors, including chromatin modifications, histone chaperones, histone variants, chromatin

Xue Xiao, Liping Dong and Yi-Zhou Wang have contributed equally to this work.

$\bowtie$ Correspondence: chenping@moon.ibp.ac.cn (P. Chen), weili007@iphy.ac.cn (W. Li) remodelers, and chromatin architectural proteins. For the genomic regions where gene expression are active, a series of epigenetic modifications (Zentner and Henikoff 2013), enzymes (Maier et al. 2000), and transcription factors (Li et al. 2016b; Pavri et al. 2006) assist in unfolding of chromatin fibers to expose DNA, allowing the transcription and replication to proceed. When the transcription or duplication is completed, the chromatin fiber needs to be reassembled (Fleming et al. 2008). Therefore, deciphering the structural dynamics and its epigenetic regulation of chromatin fibers is the basis for understanding the epigenetic regulation of gene-related biological processes including transcription and DNA replication.

Traditional biochemical methods have shown some difficulty in studying the dynamic structural changes of chromatin fibers (Fleming et al. 2008; Georgel et al. 2003). For example, the properties of chromatin fibers 
obtained in gel electrophoresis and circular dichroism are the sum of a huge number of molecules involved in the reaction; thus it is impossible to characterize the dynamic process of structural changes of chromatin fibers. Although high-resolution imaging techniques, such as X-ray and Cryo-electron microscopy (Cryo-EM), have successfully determined the high-resolution structures of the nucleosome (Luger et al. 1997) and the 30-nm chromatin fiber (Song et al. 2014), these techniques are only suitable for studying static structures and cannot monitor the dynamic structural transitions of the nucleosome and chromatin fibers. Fluorescence resonance transfer (FRET) technique can track the dynamic changes of chromatin structure at singlemolecule level (Chen et al. 2016; Fierz et al. 2011; Ngo and $\mathrm{Ha}$ 2015). However, in most time, fluorescence probe quenches in a few minutes; thus it is difficult to monitor the structural transitions in such a short-time window.

Single-molecule force spectroscopy is an ideal technique to study the structural change of biological macromolecules, especially the nucleosome and chromatin fibers. By applying different external forces on the single chromatin fiber or mononucleosome, we can manipulate conformational changes of the molecule, which could be monitored by tracking the extension of the chromatin fiber or mononucleosome. We can also deduce the stability and assembly pattern of the chromatin fiber or mononucleosome by analyzing the step size and the transition force of structural intermediates. The main techniques of single-molecular force spectroscopy include atomic force microscopy (AFM; Piontek and Roos 2018), optical tweezers (Neuman and Block 2004), and magnetic tweezers (Gosse and Croquette 2002). AFM captures biological macromolecules by a chemically modified probe and applies a pulling force, but the pulling force is usually very large and it is difficult to ensure only one single-molecule is captured. The optical tweezers require the two ends of molecule binding to two polystyrene beads respectively, and then the optical tweezers grab the polystyrene beads through the optical traps constructed by the laser, and then move the laser to apply the pulling force. Optical tweezers can achieve relatively high resolution (Abbondanzieri et al. 2005; Mahamdeh and Schäffer 2009), but it is difficult to keep constant force when tracking the conformational changes. Besides that, optical tweezers are not suitable for long time measurement, because high-intensity laser may damage the sample (Simpson et al. 1998). The magnetic tweezers connect the two ends of biological macromolecules to surface and paramagnetic bead respectively, and then apply the pulling force with an external magnetic field. Magnetic tweezers can track many biological macromolecules at the same time, but the resolution is slightly lower than optical tweezers. In this paper, we report a strategy to study the structural dynamics of chromatin fibers based on single-molecule magnetic tweezers. We modified the magnetic tweezers by adopting solid-microscope optical system, large-field objective lens, and high-speed sampling CCD camera to improve the time resolution of magnetic tweezers and the number of magnetic beads tracked at the same time. We also developed a homebuilt control software of magnetic tweezers based on LabVIEW to achieve more advanced bead tracking and data analysis algorithm, which further improves the resolution of magnetic beads position and pulling force. The chromatin fiber labeled with either biotin or digoxigenin at the two ends of DNA was reconstituted in vitro and attached to the modified magnetic tweezers. The extensions of chromatin fibers under different tension were recorded at single-molecule level to trace the dynamic structural transition. This strategy provides a new research platform to dissect the structural dynamics of chromatin fiber and its regulation by different chromatin factors.

\section{EXPERIMENTAL SECTION}

\section{Preparation of chromatin fibers}

Recombinant histones and DNA templates of 24 tandem $177 \mathrm{bp}$ repeats of the 601 sequence $(24 \times 177 \mathrm{bp})$ were cloned and purified as previously described ( $\mathrm{Li}$ et al. 2010). The $24 \times 177$ bp 601 DNA was subcloned into a modified pWM530 plasmid containing two BseYI sites flanking multiple cloning sites. The pWM530BseYI-177-24 plasmid was digested by BseYI enzyme, with the $24 \times 177$ DNA templates purified by gel extraction. The two single-stranded ends of $24 \times 177$ DNA templates were filled with either dUTP-digoxigenin or dATP-biotin by Klenow reaction at $30{ }^{\circ} \mathrm{C}$ for $3 \mathrm{~h}$. Remaining enzymes were removed by phenolic chloroform extraction and ethanol precipitation. For histone purification, pET-histone expression plasmids were transformed into BL21 (DE3) pLysS cells individually and then a single colony each was inoculated from a freshly streaked agar plate into $5 \mathrm{ml}$ of LB medium containing $100 \mu \mathrm{g} / \mathrm{mL}$ ampicillin and $25 \mu \mathrm{g} / \mathrm{mL}$ chloramphenicol. $100 \mathrm{ml}$ overnight culture from optimal clones was inoculated into 5 l LB medium and overexpression of histones were induced at $O D_{600 \mathrm{~nm}}$ of 0.4-0.6 with $0.5 \mathrm{mmol} / \mathrm{L}$ IPTG. After growing for another 3-4 h, cells were harvested by centrifugation and resuspended with buffer A $(50 \mathrm{mmol} / \mathrm{L}$ Tris- $\mathrm{HCl}$, 
pH 7.5, $100 \mathrm{mmol} / \mathrm{L} \mathrm{NaCl}, 1 \mathrm{mmol} / \mathrm{L}$ EDTA, $1 \mathrm{mmol} / \mathrm{L}$ bezamidine). After sonication and centrifugation, the pellets containing inclusion bodies of the corresponding histones were washed three times by buffer A plus $1 \%$ Triton X-100 and later washed with buffer A twice to get rid of Triton X-100. Then pellets were dissolved by gently stirring for $1 \mathrm{~h}$ at room temperature with buffer B $(7 \mathrm{~mol} / \mathrm{L}$ guanidinium $\mathrm{HCl}, 20 \mathrm{mmol} / \mathrm{L}$ Tris- $\mathrm{HCl}$, pH 7.5, $10 \mathrm{mmol} / \mathrm{L}$ DTT). With another centrifugation $(23,000 \mathrm{~g}$ for $10 \mathrm{~min})$ to remove undissolved pellets, supernatants containing unfolded histones were collected and analyzed by $15 \%$ SDS-PAGE.

The respective histone octamers were reconstituted as previously described (Dyer et al. 2004). Equimolar amounts of individual histones in unfolding buffer $\mathrm{B}$ (7 mol/L guanidinium $\mathrm{HCl}, 20 \mathrm{mmol} / \mathrm{L}$ Tris- $\mathrm{HCl}, \mathrm{pH}$ 7.5, $10 \mathrm{mmol} / \mathrm{L}$ DTT) were dialyzed into refolding buffer ( $2 \mathrm{~mol} / \mathrm{L} \mathrm{NaCl}, 10 \mathrm{mmol} / \mathrm{L}$ Tris-HCl, pH 7.5, $1 \mathrm{mmol} / \mathrm{L}$ EDTA, $5 \mathrm{mmol} / \mathrm{L}$ 2-mercaptoethanol), and purified through a Superdex S200 column. Chromatin samples were assembled using the salt-dialysis method as previously described (Song et al. 2014). The reconstitution reaction mixture with histone octamers and DNA templates in TEN buffers $(10 \mathrm{mmol} / \mathrm{L}$ Tris-HCl, $\mathrm{pH} 8.0$, $1 \mathrm{mmol} / \mathrm{L}$ EDTA, $2 \mathrm{~mol} / \mathrm{L} \mathrm{NaCl}$ ) were dialyzed for $16 \mathrm{~h}$ at $4{ }^{\circ} \mathrm{C}$ in TEN buffer, which was continuously diluted by slowly pumping in TE buffer $(10 \mathrm{mmol} / \mathrm{L}$ Tris-HCl, $\mathrm{pH}$ 8.0, $1 \mathrm{mmol} / \mathrm{L}$ EDTA) to a lower concentration of $\mathrm{NaCl}$ from 2 to $0.6 \mathrm{~mol} / \mathrm{L}$. For nucleosomal arrays, samples were collected after final dialysis in measurement HE buffer (10 mmol/L HEPES, pH 8.0, $0.1 \mathrm{mmol} / \mathrm{L}$ EDTA) for $4 \mathrm{~h}$. For histone $\mathrm{H} 1$ incorporation, an equal molar amount of histone $\mathrm{H} 1$ (relative to mono-nucleosomes) was added before the final dialysis step and further dialyzed in TE buffer with $0.6 \mathrm{~mol} / \mathrm{L} \mathrm{NaCl}$ for $3 \mathrm{~h}$. The stoichiometry of histone octamer binding to the DNA template was determined by EM investigation (Song et al. 2014).

Stoichiometry of histone octamer to DNA template and histone $\mathrm{H} 1$ to nucleosome were evaluated by EM analysis as described previously (Chen et al. 2013). The samples were fixed with $0.4 \%$ glutaraldehyde (Fluka) in HE buffer on ice for $30 \mathrm{~min}$. For metal shadowing experiment, chromatin samples were prepared in HE buffer with DNA concentrations of $5 \mu \mathrm{g} / \mathrm{mL} .2 \mathrm{mmol} / \mathrm{L}$ spermidine was added into the sample solution to enhance the absorption of chromatin to the grids. Samples were applied to the glow-discharged carboncoated EM grids and incubated for $2 \mathrm{~min}$ and then blotted. Grids were washed stepwise in $20 \mathrm{ml}$ baths of $0 \%, 25 \%, 50 \%, 75 \%$, and $100 \%$ ethanol solution for 4 min, each at room temperature, air dried and then shadowed with tungsten at an angle of $10^{\circ}$ with rotation. For negative staining studies, chromatin samples were prepared in HE buffer with DNA concentrations of $20 \mu \mathrm{g} / \mathrm{mL}$. Chromatin samples in fixative solution were incubated on glow-discharged carboncoated EM grids for $1 \mathrm{~min}$. The excess sample solution was removed using filter papers. The grid was stained in $2 \%$ uranylacetate for $2 \times 30 \mathrm{~s}$, blotted with filter papers and allowed to air-dry for several minutes. Samples were examined using a FEI Tecnai G2 Spirit $120 \mathrm{kV}$ transmission electron microscope.

\section{Setup of magnetic tweezers}

As shown in Fig. 1, the setup of magnetic tweezers is mainly composed of light source $(625 \mathrm{~nm}$ red LED, THORLABS), two NdFeB magnets with $0.5 \mathrm{~mm}$, flow cell, $60 \times$ oil immersed objective (UPLSAPO60XO, NA 1.35 , Olympus), and $1280 \times 2024$ CCD camera (MC1362, Mikrotron). The flow cell consists of a bottom coverslip, a double-sided tap with a rectangular channel $\left(5 \times 50 \mathrm{~mm}^{2}\right)$, and an upper coverslip with an inlet and outer at each end. In the flow cell, one end of the chromatin fiber is anchored to the streptavidin coated magnetic beads (M280 Invitrogen Norway), and the other end is anchored to the surface coated with antidigoxigenin. The two magnets are fixed on a highresolution translation stage (M-126, PI) and a rotation stage (C-150, PI). The exerted tension $(0-100 \mathrm{pN})$ on chromatin fibers is controlled by tuning the distance of the magnets in $z$ direction (Dammer et al. 1996; Lee et al. 1994; Merkel et al. 1999; Moy et al. 1994; Pincet and Husson 2005). At a given magnet position, the tension exerted on the chromatin samples is constant. The twist can be regulated in the twist-constrained assays by rotating the magnets. The images of beads would go through the objective and then into the CCD camera. The thermal drift is the barrier for the image tracing in a long time up to several hours. In our magnetic tweezers, four Peltier modules are applied to control the sample temperature with the resolution of $0.1{ }^{\circ} \mathrm{C}$.

\section{Coverslip cleaning and surface functionalization}

To anchor the digoxin and biotin labeled chromatin fiber on the surface, the bottom coverslip needs to be cleaned and functionalized. Firstly, several pieces of coverslips were put in a staining jar filled with deionized water and detergent, and then the staining jar was placed in an ultrasonic cleaning bath for $30 \mathrm{~min}$. After that, the coverslips were rinsed with deionized water for 5-10 times to remove the detergent. The washing process was repeated using acetone and methanol, respectively to rinse some organic impurities. To functionalize the 


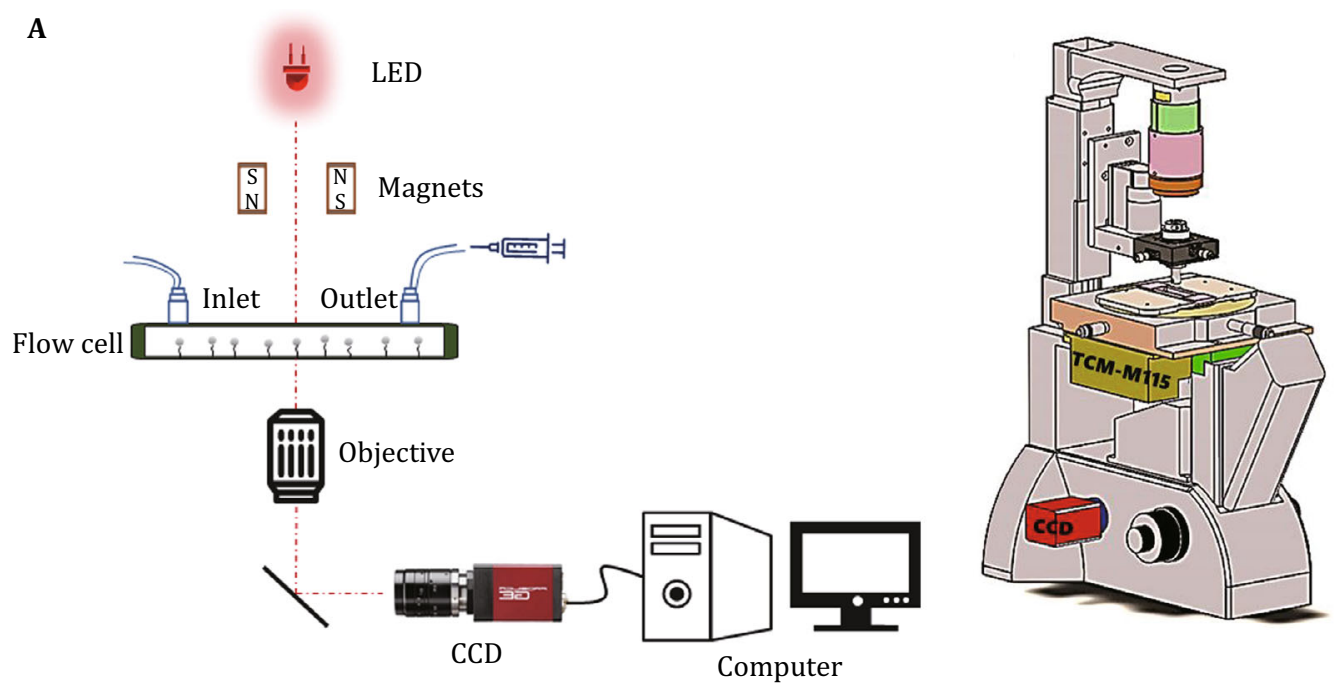

B

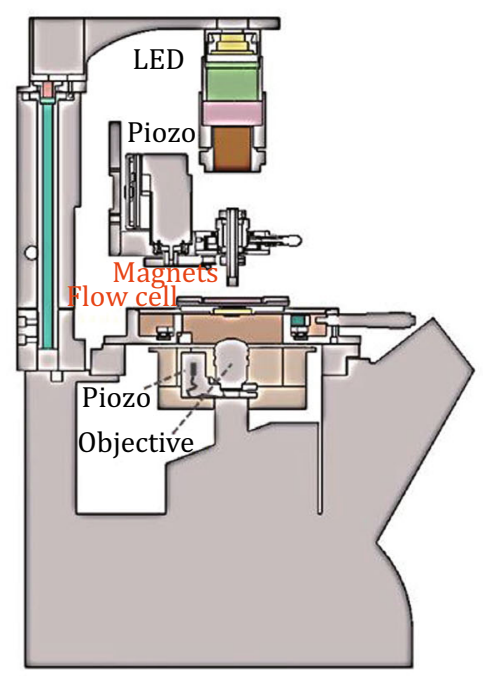

C
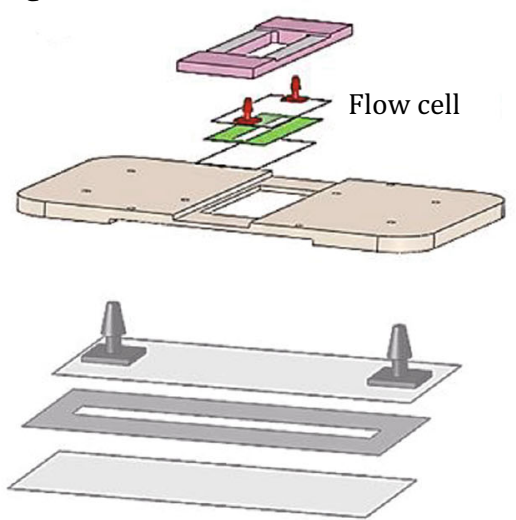

Fig. 1 Setup of magnetic tweezers. A The schematic (left) and assembly (right) diagram of magnetic tweezers. B Sectional view of magnetic tweezers. Magnetic tweezers are consisted of LED light source, a pair of magnets, a flow cell, a flow cell and a CCD camera. C The configuration of the flow cell. The flow cell is formed by two coverslips with a two-sided tap in the center

surface of coverslip, the nitrocellulose solution $(0.4 \%-$ $0.8 \% \mathrm{w} / \mathrm{v}$ ) was coated on the surface and heated at $120{ }^{\circ} \mathrm{C}$ for $3 \mathrm{~min}$, then the surface could bind with antidigoxin for tethering chromatin fibers (Cnossen et al. 2014).

To control the thermal drift, furthermore, the diluted polystyrene beads solution was dropped on the surface and heated at $\sim 150{ }^{\circ} \mathrm{C}$ for 5 min to melt on the cover-slip surface. The fixed beads were tracked simultaneously and served as the indicator of the drift. We subtracted these drift signals in our LabVIEW software.

\section{Result}

Preparation of chromatin fibers

The nucleosomal arrays and chromatin fibers used for the single-molecule magnetic tweezers investigation were reconstituted in vitro by purified histones and DNA template (Fig. 2A, B). The DNA template containing 24 tandem repeats of 177-bp Widom 601 nucleosome positioning sequence $(24 \times 177 \mathrm{bp})$ was labeled with either biotin or digoxigenin at the two ends. In the absence of linker histone, the nucleosomal arrays reconstituted on the $24 \times 177 \mathrm{bp}$ DNA templates adopted an extended beads-on-a-string conformation, as shown by EM analysis (Fig. 2C). In the presence of H1, 
Fig. 2 Preparation of chromatin samples. A SDSPAGE analysis of the purified four histones and the reconstituted histone octamers. B Agarose gel analysis of the purified $24 \times 177$ bp DNA template for magnetic tweezers. C EM (metal shadowing) images of the nucleosomal arrays without H1. D EM (negatively stained) images of the compact chromatin fibers with H1

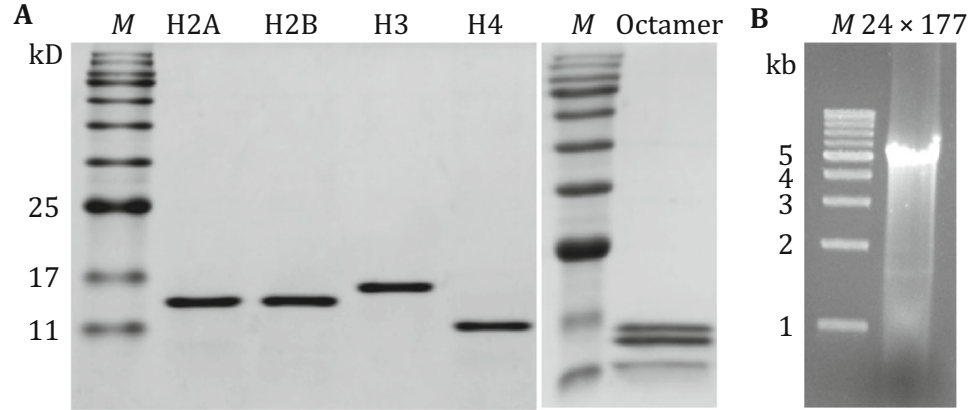

C

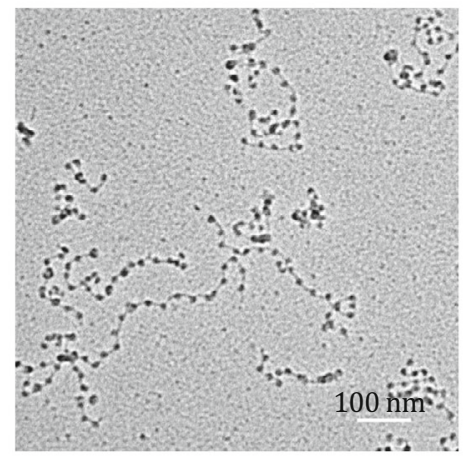

D

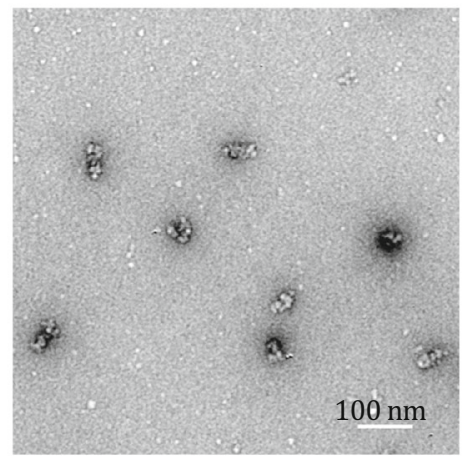

the arrays were further condensed into compact chromatin fibers (Fig. 2D).

The chromatin fiber labeled with either biotin or digoxigenin at the two ends was attached to the modified magnetic tweezers. To avoid non-specific binding of chromatin fibers to the surface and magnetic beads, the flow cell requires passivation by incubating with PBS passivation buffer $(10 \mathrm{mg} / \mathrm{mL}$ BSA, $1 \mathrm{mmol} / \mathrm{L}$ EDTA, $10 \mathrm{mmol} / \mathrm{L} \mathrm{PB}, 10 \mathrm{mg} / \mathrm{mL}$ Pluronic F127 surfactant (Sigma-Aldrich), 3 mmol/L $\mathrm{NaN}_{3}, \mathrm{pH} \mathrm{7.8)} \mathrm{for} 2 \mathrm{~h}$ (Fulconis et al. 2006; Koster et al. 2005). To tether the chromatin fiber to the surface of super paramagnetic beads (M280 Invitrogen Norway), the diluted chromatin fiber solution ( $\sim 10 \mathrm{pmol} / \mathrm{L})$ was first mixed with M280 beads for $30 \mathrm{~min}$ at room temperature on the Hula Mixer (ThermoFisher) at 1 turns/min. Secondly, passivation buffer in the flow cell was rinsed by HE buffer (10 mmol/L HEPES, $1 \mathrm{mmol} / \mathrm{L}$ EDTA) using a pump (500 $\mu \mathrm{l} / \mathrm{min})$. Thirdly, the mixture of chromatin fibers and magnetic beads was incubated in the flow cell for $10 \mathrm{~min}$ to anchor the other end of the chromatin fiber to the surface of the functionalized coverslip. At last, the unbound beads and chromatin fibers in the flow cell were subsequently washed out with $500 \mu \mathrm{l}$ HE buffer.

To identify the beads that only bind with single chromatin fiber, we pull the beads with a relatively large force $(2-3 \mathrm{pN})$ and rotate the permanent magnets $\sim 50$ turns. If the bead binds more than one chromatin fiber, the chromatin fibers would be braided and the distance of the bead to surface would decrease. On the other hand, the beads that only bind single chromatin fiber have no changes in distance to surface, which could be tracked in further stretching experiments.

\section{Tracking the magnetic beads in three-dimensions}

In stretching experiments, the extension of chromatin fibers is determined by tracking the three-dimensional (3D) position of the magnetic beads. The positions of a magnetic bead in the horizontal direction $(X, Y)$ can be determined by calculating the center of its diffraction pattern. A common bead tracking method is onedimensional (1D) cross-correlation algorithm (Gosse and Croquette 2002), but the accuracy is relatively low. Our magnetic tweezers use the quadrant interpolation algorithm developed by Cees Dekker (van Loenhout et al. 2012), which is an improved version of conventional 1D cross-correlation algorithm. In brief, we calculate the estimated center $\left(X_{0}, Y_{0}\right)$ of a bead using a center-of-mass computation, which is shown as follow:

$X_{0}=\frac{\sum_{i=1}^{N} \sum_{j=1}^{N}\left(i I_{i j}\right)}{\sum_{i=1}^{N} \sum_{j=1}^{N} I_{i j}}, \quad Y_{0}=\frac{\sum_{i=1}^{N} \sum_{j=1}^{N}\left(j I_{i j}\right)}{\sum_{i=1}^{N} \sum_{j=1}^{N} I_{i j}}$,

where $i$ and $j$ are pixel subscripts of extracted bead image and $I_{i j}$ represents the light intensity of the $j$ th 
pixel point of the $i$ th line. Based on the estimated center, the image is divided into four quadrants to sample radial profiles at polar coordinates, respectively. When calculating the $X$ position of a bead, the sum of right radial profiles is concatenated with the left profiles, thus creating an intensity profile $I_{x}(r)$ along the $x$ direction. $I_{x}(r)$ is cross-correlated with its mirror profile $I_{x}(-r)$ :

$C_{X x}=\operatorname{IFFT}\left(\operatorname{FFT}\left(I_{x}(r)\right) * \overline{\operatorname{FFT}\left(I_{x}(-r)\right)}\right)$,

where FFT is fast Fourier transform and IFFT is inverse fast Fourier transform, $C_{X X}$ is resulting cross correlative curve. The $X$ position of bead can be derived from the peak position $d_{r}$ of resulting cross correlative curve $C_{X X}$ as follow:

$X=X_{0}+\frac{2 d_{r}}{\pi}$,

where $X_{0}$ is $X$ coordinate of estimated center, $\pi$ is circumference rate. By creating the intensity profile along the $Y$ direction, we could calculate the $Y$ position accordingly.

Tracking the bead position in vertical direction $(z$ direction) requires a prerequisite calibration that building a relationship between defocused distance and diffraction pattern of beads (Gosse and Croquette 2002). Firstly, by moving magnets toward flow cell, a relatively large force $(\sim 2-3 \mathrm{pN})$ is applied on beads to reduce the fluctuation of beads. Then the inversed objective is moving at $\sim 100 \mathrm{~nm}$ step size, while the CCD is recording the diffraction pattern at every step (Fig. 3A-D). The relationship between diffraction patterns and defocused distance can create a twodimensional (2D) look-up table (LUT) through bi-linear
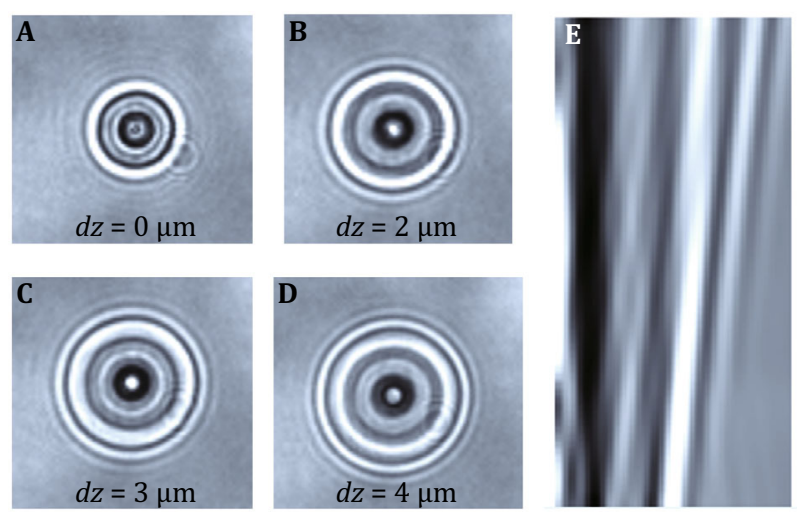

Fig. 3 Generation of a calibration profile in the $z$ direction for a bead. A-D The diffraction patterns of the bead with various distances to the focal plane. E By recording the size and density of the diffraction rings at different distance to the focal plane, a generated calibration profile is used to measure vertical relative displacements of the bead interpolation (Fig. 3E). To determine the $Z$ position of a given image, the $\chi^{2}$ value of the radial profile $I(r)$ with LUT is calculated:

$\chi^{2}=\sum_{r=0}^{R}\left(I(r)-I_{\mathrm{LUT}}(r)\right)^{2}$.

Thus, the minimum $\chi^{2}$ corresponds to the $Z$ position of the image.

\section{Calculation of applied force}

To calculate force applied on samples, a common used method is treating the bead-chromatin system as an inversed pendulum with small displacements in the vicinity equilibrium position (Klaue and Seidel 2009; Strick et al. 1998). In this model, the magnetic bead is attached to a rigid rod with length $l$, and the force $F_{\text {mag }}$ exerts on it. When the pendulum has a small displacement $a$, restoring force is giving by $F_{a}=-\frac{F_{\text {mag }}}{l} \times a$. This equation is equivalent to the rule of a Hookean spring with the spring constant $k_{a}=\frac{F_{\text {mag }}}{l}$, according the equipartition theorem,

$E_{\text {therm }}=\frac{1}{2} k_{a}\left\langle a^{2}\right\rangle=\frac{1}{2} k_{\mathrm{B}} T$,

where $k_{\mathrm{B}}$ is Boltzmann constant and $T$ is temperature. Then we get the force $F_{\text {mag: }}$

$F_{\text {mag }}=\frac{k_{\mathrm{B}} T}{\left\langle a^{2}\right\rangle} l$,

where $\left\langle a^{2}\right\rangle$ is the variance of bead in lateral fluctuation, and $l$ is effective pendulum length, namely the sum of radius of bead and extension of chromatin fiber. Therefore, the applied force can be determined from the lateral fluctuation of the bead (Fig. 4A, B). However, in practice, at forces greater than $\sim 10 \mathrm{pN}$, the measurement of $\left\langle a^{2}\right\rangle$ in real space appears systematic error because of the finite camera acquisition frequency. Therefore, power spectral density (PSD) analysis is typically used to analyze the in-plane fluctuations.

Considering the viscous drag coefficient $\gamma_{a}$, the PSD can be expressed:

$S_{a}(f)=\frac{4 k_{\mathrm{B}} T \gamma_{a}}{k_{a}^{2}} \frac{1}{1+\left(f / f_{\mathrm{c}}\right)^{2}}$,

where $f$ is the frequency and $f_{\mathrm{c}}=\frac{k_{a}}{2 \pi \gamma_{a}}$ is characteristic cutoff frequency. Due to the limiting of acquisition frequency of camera $f_{\mathrm{s}}$, low-pass filtering and aliasing should be considered (Klaue and Seidel 2009; Lansdorp and Saleh 2012; te Velthuis et al. 2010) as follows, 
A
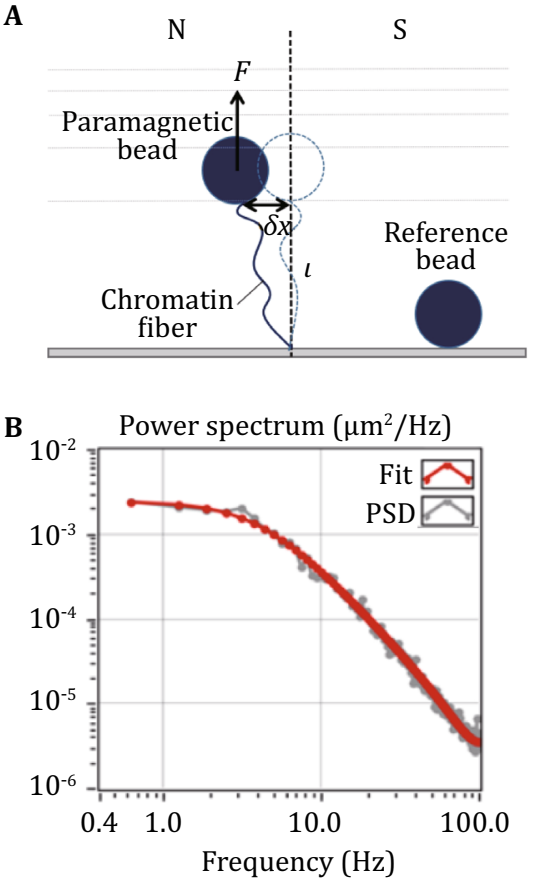

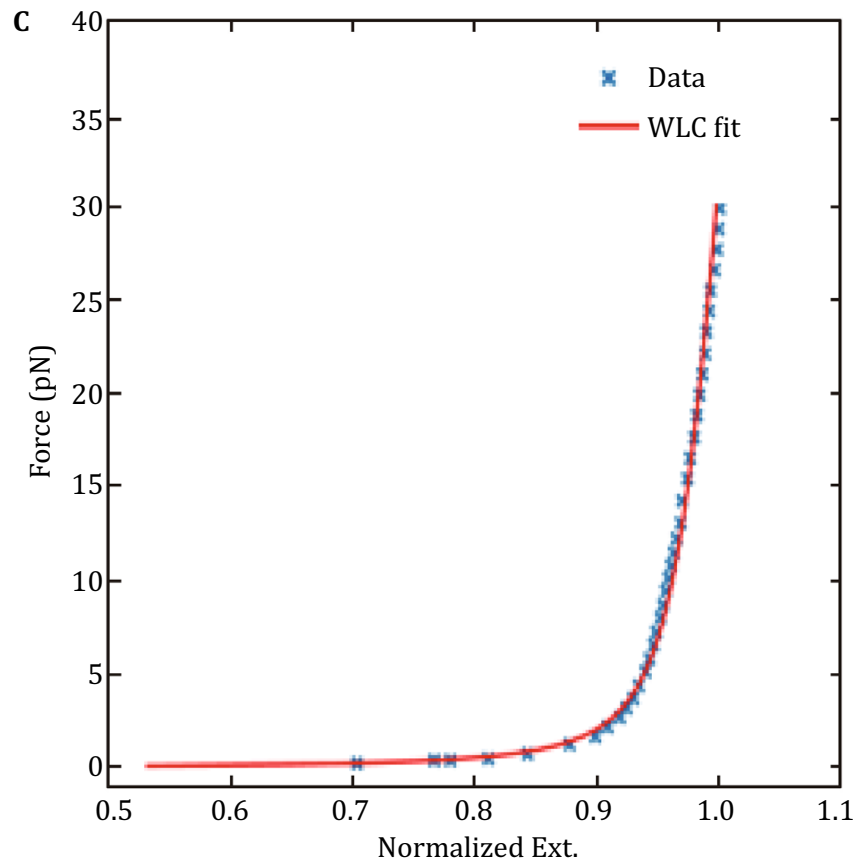

Fig. 4 The force calculation for the DNA tether. A A schematic representation of a tethered bead with a reference bead fixed to the bottom coverslip. The exerted tension $F$ on the paramagnetic bead arises from the gradient of the magnetic field. B The spectrum and the fitted curve of the one-dimensional fluctuations of the paramagnetic bead under a constant tension. C For a $10 \mathrm{~kb}$ dsDNA, the relationship between the measured force and extension is consistent with the WLC (worm-like chain) model

$S_{a}^{\text {corr }}(f)=\sum_{n=-\infty}^{\infty} \frac{4 k_{\mathrm{B}} T \gamma_{a}}{k_{a}^{2}} \frac{1}{1+\left(\left|f+n f_{\mathrm{s}}\right| / f_{\mathrm{c}}\right)^{2}} \frac{\sin ^{2}\left(\pi \tau_{\mathrm{e}}\left|f+n f_{\mathrm{s}}\right|\right)}{\left(\pi \tau_{\mathrm{e}}\left|f+n f_{\mathrm{s}}\right|\right)^{2}}$

where $\tau_{\mathrm{e}}$ is the exposure time of the camera. This formula can be used to fit the PSD, obtaining the $k_{a}$, then the $F_{\text {mag. }}$ In our experiment, we used an improved PSD fitting method developed by Ralf Seidel, which takes into account the exposure time, direction of magnet field and rotation of bead (Daldrop et al. 2015).

To calibrate the force derived from our magnetic tweezers, we stretched a dsDNA with a length of $10 \mathrm{~kb}$, then we derived the applied force from lateral fluctuation of beads and fitted with an extensible WLC model (Odijk 1995):

$\frac{z}{L}=1-\frac{1}{2}\left(\frac{k_{\mathrm{B}} T}{F L_{\mathrm{P}}}\right)^{1 / 2}+\frac{F}{S}$

where $z$ and $L$ are extension and the contour length of molecule, $L_{\mathrm{P}}$ is persistence length that characterize the rigidity of molecule, and $S$ is stretch modulus of the molecule. As shown in Fig. 4C, our experiment result agrees well with the WLC model, indicating a good performance of our magnetic tweezers.

\section{Structural dynamics of chromatin fibers revealed by magnetic tweezers}

Using our improved magnetic tweezers, we investigated the hierarchical organization and structural dynamics of chromatin fibers reconstituted in vitro in the presence of H1 (Fig. 5A), whose 3D Cryo-EM structures have been resolved recently (Li et al. 2016c; Song et al. 2014). To trace the structural transition, we applied a continuously increasing force on a single chromatin fiber by moving the magnets in $z$ direction at $2 \mu \mathrm{m} / \mathrm{s}$. The forceextension curve of a chromatin fiber containing $\mathrm{H} 1$ is shown by the blue curve, with that of a nucleosomal array without $\mathrm{H} 1$ for comparison (Fig. 5B). At force above $5 \mathrm{pN}$, similar stepwise unfolding events were observed for both extended nucleosomal arrays and compacted chromatin fibers, which correspond to the rupture states of nucleosomes in the chromatin fiber (Bintu et al. 2012; Brower-Toland et al. 2002; Hall et al. 2009). At low-force region $(<5 \mathrm{pN})$, the curve of the chromatin fiber begins to deviate from that of the nucleosomal array at $\sim 1 \mathrm{pN}$, and a distinct force plateau spanning a range of $\sim 100 \mathrm{~nm}$ near $3 \mathrm{pN}$ is clearly identified (Fig. 5B). The structural transition near $3 \mathrm{pN}$ should be attributed to the disruption of 
A

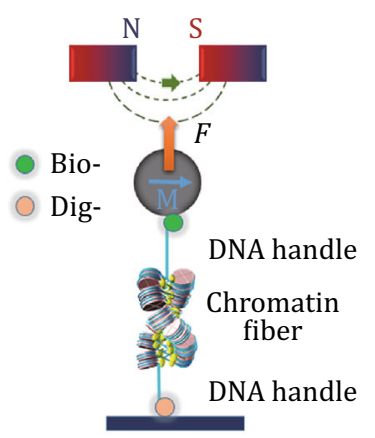

B

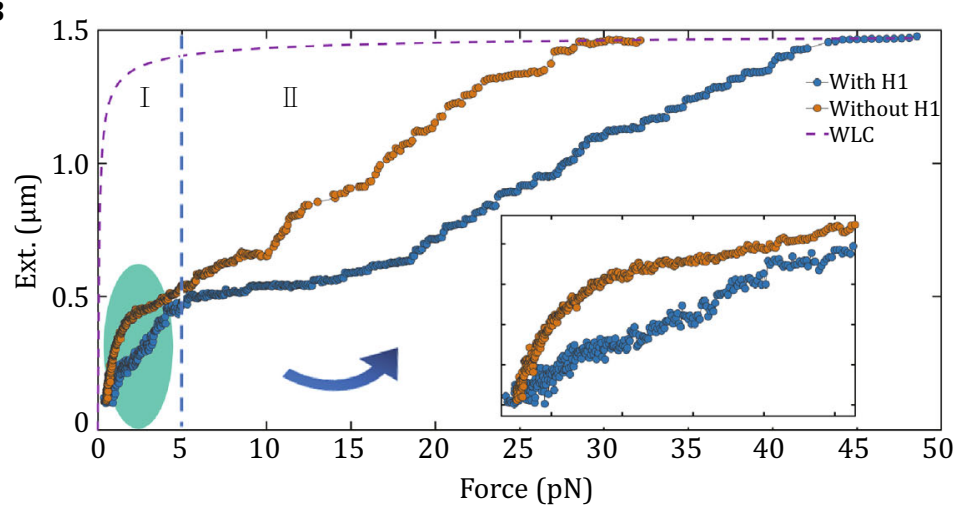

C

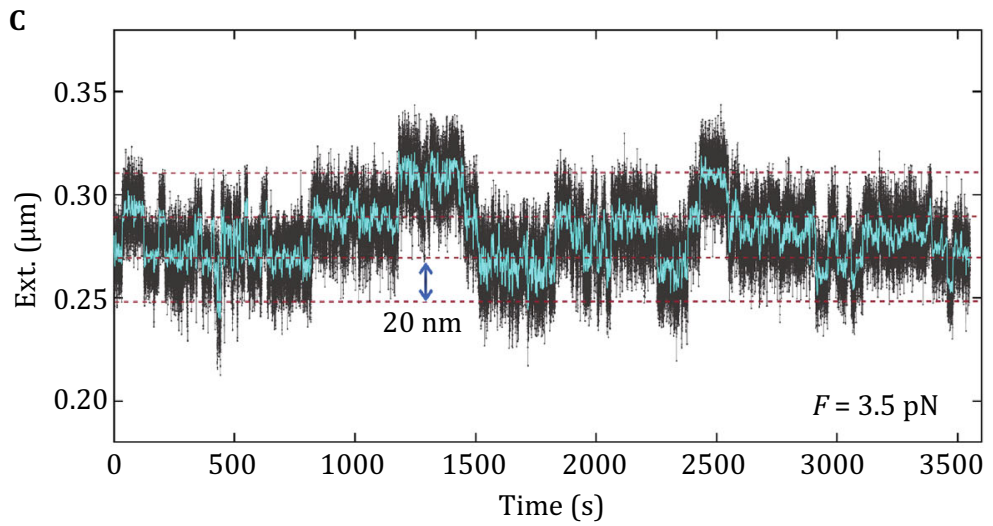

E

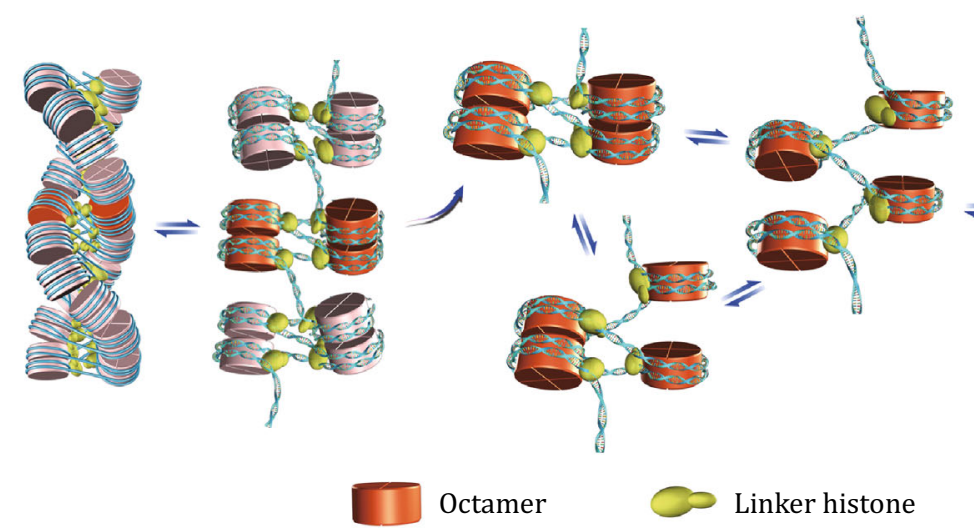

D

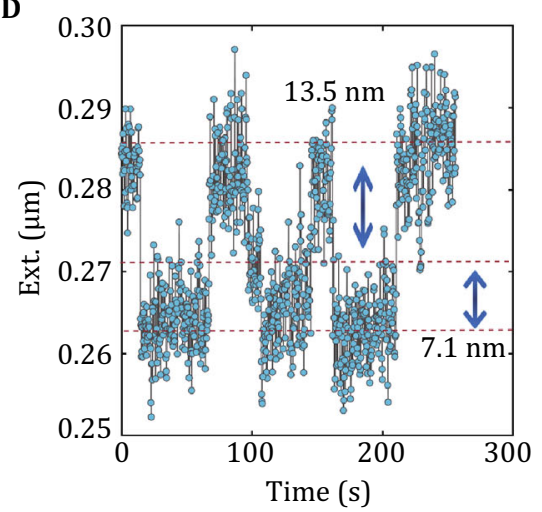

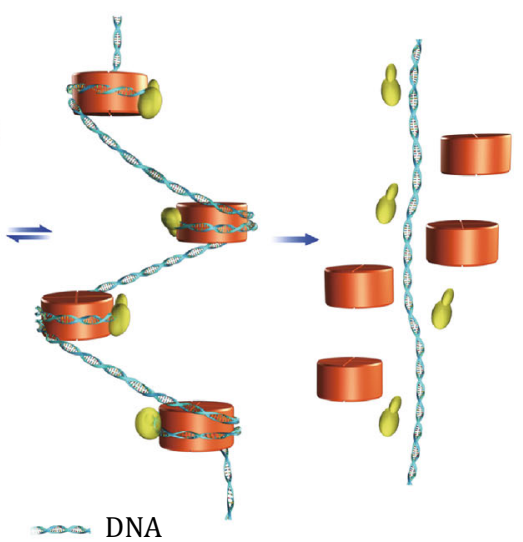

Fig. 5 Investigation of structural dynamics of chromatin fibers by single-molecule magnetic tweezers. A Schematic representation of chromatin fiber studied by magnetic tweezers. B Comparison of two typical force-extension curves of a chromatin fiber with H1 (blue curve) and without $\mathrm{H} 1$ (orange curve) in $\mathrm{HE}$ buffer, with two major stages at high force $(>5 \mathrm{pN})$ or low force $(<5 \mathrm{pN})$ recognized. The inset shows the details of stage at low force $(<5 \mathrm{pN})$. C Stepwise folding and unfolding dynamics of tetranucleosomal units at $3.5 \mathrm{pN}$ for the chromatin fiber with H1. D Stepwise folding and unfolding dynamics of each tetranucleosomal unit with two alternative pathways at $3.5 \mathrm{pN}$ for the chromatin fiber with H1. E Model for the dynamic organization of chromatin fiber. The left-handed double-helix chromatin fiber unfolds to a "tetranucleosomes-on-a string" extended structure, and then unfolds to a complete open nucleosomal array in one or two steps

nucleosome-nucleosome interactions in the higherorder chromatins, as the nucleosomes remain intact at such low forces.
In order to gain more insights into the nature of structural transition near $3 \mathrm{pN}$, we performed the forceclamp measurements of chromatin fibers. Interestingly, 
a reversible folding and unfolding dynamical behavior was observed at $3.5 \mathrm{pN}$ (Fig. 5C), with the obvious transition step size of $\sim 20 \mathrm{~nm}$. The details of the folding and unfolding dynamics of chromatin fiber at $3.5 \mathrm{pN}$ indicate that the structure transition with step of $20 \mathrm{~nm}$ is always accomplished within two steps, which corresponds to the two-step disruption of tetranucleosome structural unit (Fig. 5D). Importantly, similar dynamic processes can be observed in the chromatin fibers assembled not only on regular tandem repeat of 601 DNA sequence but also on the scrambled (non-repetitive) DNA sequence, suggesting that the existence of tetra-nucleosome units is not dependent on DNA sequence. By using the modified single-molecule magnetic tweezers method, we show that the tetranucleosome unit exists as a stable structural intermediate of the 30-nm chromatin fiber and further unfolds to a more extended "beads-on-a-string" conformation by disrupting the nucleosome-nucleosome interactions within tetranucleosomal unit (Fig. 5E) (Li et al. 2016b).

\section{DISCUSSION}

In eukaryotic gene activation, the compact chromatin fibers must unfold for transcription and other DNArelated biological processes. Chromatin dynamics is critical to regulate the accessibility of transcription factors via dynamic transitions between the compact chromatin fiber and the more accessible nucleosomal array in vivo (Li et al. 2010; Li and Reinberg 2011). Therefore, it is of great importance to study how the dynamic organization of the chromatin fiber is regulated. However, there are various technical challenges to probe the detailed structure and the dynamics of chromatin fiber using traditional biochemical assays (Vilfan et al. 2009). For example, static conformations obtained by Cryo-EM structural study in vitro cannot provide much detailed information for dynamic processes. As a single-molecule method, magnetic tweezers is a powerful tool to study the dynamic organization of chromatin fibers by tracing the real-time folding and unfolding of single chromatin fiber (Bintu et al. 2012; Brower-Toland et al. 2002; Hall et al. 2009; Kruithof et al. 2009; Meng et al. 2015; Yan et al. 2007).

In this paper, we present a platform for dissecting dynamics of chromatin fibers using single-molecule magnetic tweezers. With more advanced tracking algorithms and higher resolution, we can observe the unfolding details of chromatin fibers and monitor the folding dynamics of chromatin fibers by careful tension adjustment. With magnetic tweezers, we have revealed that tetra-nucleosome unit exists as a stable structural intermediate during the formation of $30-\mathrm{nm}$ chromatin fiber. These results are the complement of traditional biochemical experiments and high-resolution static imaging techniques such as EM, depicting the dynamics of single chromatin fiber, and providing a new perspective for understanding the dynamic organization of chromatin fibers and its regulation by chromatin factors.

Magnetic tweezers are widely used in the study of biological macromolecules because of their simple principle, low cost, high parallelism and single-molecule tracking. In addition to studying the mechanical properties of DNA and its higher-order structures, magnetic tweezers can also be used to study the folding kinetics of various proteins, such as actin (Yuan et al. 2017) and trans-membrane proteins (Min et al. 2015). The constant force measurement, which is easy to be realized by magnetic tweezers, is also suitable for studying the working mechanism of DNA-related enzymes, such as condensin (Eeftens et al. 2017), helicase (Li et al. 2016a; Seol et al. 2016), and polymerase (Maier et al. 2000).

In the last 20 years, the technology and analytical theory of magnetic tweezers have been continuously improved. According to the needs of experiments, a series of improvements on magnetic tweezers have been made. For example, Yan Jie's group developed the transverse magnetic tweezers, which can greatly improve the force applied by the magnetic beads (Yan et al. 2004). They also developed a non-disturbing buffer exchange technology to replace the buffer environment in the measurement process (Le et al. 2015). In order to improve the resolution of magnetic tweezers, Ralf Seidel et al. used CMOS camera with ultra-high sampling rate and image processing technology base on GPU, improving the resolution of magnetic tweezers to angstrom level (Huhle et al. 2015). On the other hand, Terence R. Strick et al. used single-molecule FRET technology to combine with magnetic tweezers (Graves et al. 2015), which not only can improve the resolution of length change, but also can be used to reveal whether the protein falls off in the process of a structural transformation. Further development of these technologies may provide new platform to solve the hard questions in life science.

Acknowledgements This work was supported by Grants from the National Natural Science Foundation of China (11474346, 31471218, 31630041, 31525013, 31521002 and 61275192), the Key Research Program of Frontier Sciences, CAS (QYZDB-SSWSLH045, QYZDY-SSW-SMC020), the Youth Innovation Promotion Association CAS (2015071), the Ministry of Science and Technology of China (2017YFA0504200, 2015CB856200), (HHMI International Research Scholar Grant (55008737),and the National Key Research and Development Program (2016YFA0301500). 


\section{Compliance with Ethical Standards}

Conflict of interest Xue Xiao, Liping Dong, Yi-Zhou Wang, PengYe Wang, Ming Li, Guohong Li, Ping Chen and Wei Li declare that they have no conflict of interest.

Human and animal rights and informed consent This article does not contain any studies with human or animal subjects performed by any of the authors.

Open Access This article is distributed under the terms of the Creative Commons Attribution 4.0 International License (http:// creativecommons.org/licenses/by/4.0/), which permits unrestricted use, distribution, and reproduction in any medium, provided you give appropriate credit to the original author(s) and the source, provide a link to the Creative Commons license, and indicate if changes were made.

\section{References}

Abbondanzieri EA, Greenleaf WJ, Shaevitz JW, Landick R, Block SM (2005) Direct observation of base-pair stepping by RNA polymerase. Nature 438:460-465

Bickmore WA, van Steensel B (2013) Genome architecture: domain organization of interphase chromosomes. Cell 152:1270-1284

Bintu L, Ishibashi T, Dangkulwanich M, Wu Y-Y, Lubkowska L, Kashlev M, Bustamante C (2012) Nucleosomal elements that control the topography of the barrier to transcription. Cell 151:738-749

Brower-Toland BD, Smith CL, Yeh RC, Lis JT, Peterson CL, Wang MD (2002) Mechanical disruption of individual nucleosomes reveals a reversible multistage release of DNA. Proc Natl Acad Sci USA 99:1960

Chen P, Zhao J, Wang Y, Wang M, Long H, Liang D, Huang L, Wen Z, Li W, Li X, Feng H, Zhao H, Zhu P, Li M, Wang QF, Li G (2013) H3.3 actively marks enhancers and primes gene transcription via opening higher-ordered chromatin. Genes Dev 27:2109-2124

Chen Y, Tokuda JM, Topping T, Meisburger SP, Pabit SA, Gloss LM, Pollack L (2016) Asymmetric unwrapping of nucleosomal DNA propagates asymmetric opening and dissociation of the histone core. Proc Natl Acad Sci USA. https://doi.org/10. 1073/pnas.1611118114

Cnossen JP, Dulin D, Dekker NH (2014) An optimized software framework for real-time, high-throughput tracking of spherical beads. Rev Sci Instrum. https://doi.org/10.1063/1. 4898178

Daldrop P, Brutzer H, Huhle A, Kauert DJ, Seidel R (2015) Extending the range for force calibration in magnetic tweezers. Biophys J 108:2550-2561

Dammer U, Hegner M, Anselmetti D, Wagner P, Dreier M, Huber W, Güntherodt HJ (1996) Specific antigen/antibody interactions measured by force microscopy. Biophys J 70:2437-2441

Dyer PN, Edayathumangalam RS, White CL, Bao Y, Chakravarthy S, Muthurajan UM, Luger K (2004) Reconstitution of nucleosome core particles from recombinant histones and DNA. Methods Enzymol 375:23-44

Eeftens JM, Bisht S, Kerssemakers J, Kschonsak M, Haering CH, Dekker C (2017) Real-time detection of condensin-driven DNA compaction reveals a multistep binding mechanism. EMBO J. https://doi.org/10.15252/embj.201797596
Fierz B, Chatterjee C, McGinty RK, Bar-Dagan M, Raleigh DP, Muir TW (2011) Histone H2B ubiquitylation disrupts local and higher-order chromatin compaction. Nat Chem Biol 7:113-119

Fleming AB, Kao C-F, Hillyer C, Pikaart M, Osley M (2008) H2B ubiquitylation plays a role in nucleosome dynamics during transcription elongation. Mol Cell 31:57-66

Fulconis R, Mine J, Bancaud A, Dutreix M, Viovy J-L (2006) Mechanism of RecA-mediated homologous recombination revisited by single molecule nanomanipulation. EMBO J 25:4293-4304

Georgel PT, Horowitz-Scherer RA, Adkins N, Woodcock CL, Wade PA, Hansen JC (2003) Chromatin compaction by human $\mathrm{MeCP} 2$. Assembly of novel secondary chromatin structures in the absence of DNA methylation. J Biol Chem 278:32181-32188

Gosse C, Croquette V (2002) Magnetic tweezers: micromanipulation and force measurement at the molecular level. Biophys J 82:3314-3329

Graves ET, Duboc C, Fan J, Stransky F, Leroux-Coyau M, Strick TR (2015) A dynamic DNA-repair complex observed by correlative single-molecule nanomanipulation and fluorescence. Nat Struct Mol Biol 22:452-457

Hall MA, Shundrovsky A, Bai L, Fulbright RM, Lis JT, Wang MD (2009) High-resolution dynamic mapping of histone-DNA interactions in a nucleosome. Nat Struct Mol Biol 16:124

Huhle A, Klaue D, Brutzer H, Daldrop P, Joo S, Otto O, Keyser UF, Seidel R (2015) Camera-based three-dimensional real-time particle tracking at kHz rates and Ångström accuracy. Nat Commun 6:5885

Klaue D, Seidel R (2009) Torsional stiffness of single superparamagnetic microspheres in an external magnetic field. Phys Rev Lett 102:028302

Koster DA, Croquette V, Dekker C, Shuman S, Dekker NH (2005) Friction and torque govern the relaxation of DNA supercoils by eukaryotic topoisomerase IB. Nature 434:671

Kruithof M, Chien F-T, Routh A, Logie C, Rhodes D, van Noort J (2009) Single-molecule force spectroscopy reveals a highly compliant helical folding for the $30-\mathrm{nm}$ chromatin fiber. Nat Struct Mol Biol 16:534

Lansdorp BM, Saleh OA (2012) Power spectrum and Allan variance methods for calibrating single-molecule videotracking instruments. Rev Sci Instrum 83:025115

Le S, Yao M, Chen J, Efremov AK, Azimi S, Yan J (2015) Disturbance-free rapid solution exchange for magnetic tweezers single-molecule studies. Nucleic Acids Res. https://doi.org/10.1093/nar/gkv554

Lee GU, Kidwell DA, Colton RJ (1994) Sensing discrete streptavidinbiotin interactions with atomic force microscopy. Langmuir 10:354-357

Li G, Reinberg D (2011) Chromatin higher-order structures and gene regulation. Curr Opin Genet Dev 21:175-186

Li G, Margueron R, Hu G, Stokes D, Wang Y-H, Reinberg D (2010) Highly compacted chromatin formed in vitro reflects the dynamics of transcription activation in vivo. Mol Cell 38:41-53

Li JH, Lin WX, Zhang B, Nong DG, Ju HP, Ma JB, Xu CH, Ye FF, Xi XG, Li M, Lu Y, Dou SX (2016a) Pif1 is a force-regulated helicase. Nucleic Acids Res. https://doi.org/10.1093/nar/gkw295

Li W, Chen P, Yu J, Dong L, Liang D, Feng J, Yan J, Wang P-YY, Li Q Zhang Z, Li M, Li G (2016b) FACT remodels the tetranucleosomal unit of chromatin fibers for gene transcription. Mol Cell 64:120-133

Li X, Liu H, Cheng L (2016c) Symmetry-mismatch reconstruction of genomes and associated proteins within icosahedral viruses using cryo-EM. Biophys Rep 2:25-32 
Luger K, Mäder AW, Richmond RK, Sargent DF, Richmond TJ (1997) Crystal structure of the nucleosome core particle at $2.8 \AA$ resolution. Nature 389:251-260

Mahamdeh M, Schäffer E (2009) Optical tweezers with millikelvin precision of temperature-controlled objectives and base-pair resolution. Opt Express 17:17190-17199

Maier B, Bensimon D, Croquette V (2000) Replication by a single DNA polymerase of a stretched single-stranded DNA. Proc Natl Acad Sci USA 97:12002-12007

Meng H, Andresen K, van Noort J (2015) Quantitative analysis of single-molecule force spectroscopy on folded chromatin fibers. Nucleic Acids Res 43:3578-3590

Merkel R, Nassoy P, Leung A, Ritchie K, Evans E (1999) Energy landscapes of receptor-ligand bonds explored with dynamic force spectroscopy. Nature 397:50

Min D, Jefferson RE, Bowie JU, Yoon T-YY (2015) Mapping the energy landscape for second-stage folding of a single membrane protein. Nat Chem Biol. https://doi.org/10.1038/ nchembio.1939

Moy VT, Florin EL, Gaub HE (1994) Intermolecular forces and energies between ligands and receptors. Science 266:257

Neuman KC, Block SM (2004) Optical trapping. Rev Sci Instrum 75:2787-2809

Ngo TTM, Ha T (2015) Nucleosomes undergo slow spontaneous gaping. Nucleic Acids Res. https://doi.org/10.1093/nar/ gkv276

Odijk T (1995) Stiff chains and filaments under tension. Macromolecules 28:7016-7018

Pavri R, Zhu B, Li G, Trojer P, Mandal S, Shilatifard A, Reinberg D (2006) Histone H2B monoubiquitination functions cooperatively with FACT to regulate elongation by RNA polymerase II. Cell 125:703-717

Pincet F, Husson J (2005) The solution to the streptavidin-biotin paradox: the influence of history on the strength of single molecular bonds. Biophys J 89:4374-4381

Piontek MC, Roos WH (2018) Atomic force microscopy: an introduction. Methods Mol Biol (Clifton NJ) 1665:243-258

Richmond TJ, Davey CA (2003) The structure of DNA in the nucleosome core. Nature 423:145-150
Seol Y, Strub M-PP, Neuman KC (2016) Single molecule measurements of DNA helicase activity with magnetic tweezers and $t$ test based step-finding analysis. Methods (S Diego Calif) 105:119-127

Simpson NB, McGloin D, Dholakia K, Allen L, Padgett MJ (1998) Optical tweezers with increased axial trapping efficiency. J Mod Opt 45:1943-1949

Song F, Chen P, Sun D, Wang M, Dong L, Liang D, Xu R-MM, Zhu P, Li G (2014) Cryo-EM study of the chromatin fiber reveals a double helix twisted by tetranucleosomal units. Science (NY NY) 344:376-380

Strick TR, Croquette V, Bensimon D (1998) Homologous pairing in stretched supercoiled DNA. Proc Natl Acad Sci USA 95:10579-10583

te Velthuis AJW, Kerssemakers JWJ, Lipfert J, Dekker NH (2010) Quantitative guidelines for force calibration through spectral analysis of magnetic tweezers data. Biophys J 99:1292-1302

van Loenhout MT, Kerssemakers JW, De Vlaminck I, Dekker C (2012) Non-bias-limited tracking of spherical particles, enabling nanometer resolution at low magnification. Biophys J 102:2362-2371

Vilfan ID, Lipfert J, Koster DA, Lemay SG, Dekker NH (2009) Magnetic tweezers for single-molecule experiments. In: Hinterdorfer P, Oijen A (eds) Handbook of single-molecule biophysics. Springer, New York, pp 371-395

Yan J, Skoko D, Marko JF (2004) Near-field-magnetic-tweezer manipulation of single DNA molecules. Phys Rev E 70:011905

Yan J, Maresca TJ, Skoko D, Adams CD, Xiao B, Christensen MO, Heald R, Marko JF (2007) Micromanipulation studies of chromatin fibers in Xenopus egg extracts reveal ATPdependent chromatin assembly dynamics. Mol Biol Cell 18:464-474

Yuan G, Le S, Yao M, Qian H, Zhou X, Yan J, Chen H (2017) Elasticity of the transition state leading to an unexpected mechanical stabilization of titin immunoglobulin domains. Angew Chem Int Ed 56:5490-5493

Zentner GE, Henikoff S (2013) Regulation of nucleosome dynamics by histone modifications. Nat Struct Mol Biol 20:259-266 